\title{
COVID-19: No olvidemos los fundamentos
}

\section{COVID-19: Do not forget the basics}

Eduardo Kattan, MD, MMEd¹, Guillermo Bugedo, MD¹

\footnotetext{
L
} a humanidad ha sabido permanentemente de guerras, hambrunas y epidemias que tienden a diezmar su población. Avances sociales, científicos y tecnológicos nos han permitido lograr períodos de paz y tranquilidad en que esas catástrofes son solo temas de libros y películas. Sin embargo, cada cierto tiempo, las epidemias o pandemias vuelven a atacar y nos sentimos débiles, vulnerables, recordándonos nuestra limitada condición humana. La peste bubónica en el siglo XIV diezmó la población europea en ese entonces, mientras la gripe española, hace 100 años, cobró entre 20 a 50 millones de vidas, entre las más recordadas.

Hace 10 años, la gripe porcina, probablemente la misma cepa de influenza H1N1 de la gripe española, mató miles de personas a nivel mundial. En Chile, hacia junio de 2009 las Unidades de Pacientes Críticos se llenaban de pacientes con neumonía que requerían soporte ventilatorio por largo tiempo. Así, copamos el parque de ventiladores, debiendo ventilar con equipos de transporte y máquinas de anestesia. Sin embargo, más crítico aún, fue la falta de profesionales capacitados (en todos los estamentos) y de protocolos de atención para ofrecer la mejor atención a estos enfermos. Tomamos malas decisiones y gastos absurdos buscando la bala mágica que derrotaría la enfermedad. En vez de aplicar los principios fisiológicos básicos de la ventilación protectora, se compró osciladores de alta frecuencia (equipos caros, ruidosos, obsoletos y difíciles de programar), que más tarde mostrarían que aumentan la mortalidad[1],[2]. Si solo hubiéramos ventilado a esos pacientes con $6 \mathrm{ml} / \mathrm{kg}$ de peso ideal (IBW), PEEP 10 y prono, nuestros resultados habrían sido diferentes.

Hoy en día, la nueva pandemia COVID-19 vuelve a recordarnos nuestra frágil condición humana, y aún no sabemos hasta cuándo y dónde llegará[3],[4]. Recordando las lecciones de hace 10 años, lo más importante es cuidar el recurso humano y hacer aquello que ha demostrado impactar los resultados y a costos racionales. Esto significa disponer de equipos de protección personal (EPP), así como ser proactivos en prevenir el desgaste físico y emocional de quienes trabajamos en los servicios de urgencia y cuidado intensivos.

Desde el punto vista de infraestructura y equipamiento, es una amenaza el número de pacientes que puedan requerir soporte ventilatorio, y de ahí la importancia para la población de evitar el contacto social y quedarse en casa. El parque de ventiladores se va a ver estresado al máximo. No requerimos más equipos de oxigenación por circulación extracorpórea (ECMO)[5], sino más ventiladores mecánicos confiables y personal capacitado que los sepa utilizar. Los anestesistas somos la segunda línea de defensa, y tendremos un rol clave en la conducción y cuidado de muchos pacientes críticos

En la era de las redes sociales, la hiperconectividad, y los "fake news", la sobreinformación es otro riesgo. Diariamente nos bombardean en nuestros teléfonos móviles con megabytes de información, audios, y recomendaciones de "expertos", sin autoría, o

Departamento de Medicina Intensiva, Facultad de Medicina, Pontificia Universidad Católica de Chile. Santiago, Chile.

Fecha de recepción: 27 de marzo de 2020

Fecha de aceptación: 27 de marzo de 2020

\section{ORCID}

https://orcid.org/0000-0001-7527-6202

Correspondencia:

Eduardo Kattan

Guillermo Bugedo

Email: e.kattan@gmail.com

Email: gbugedo@gmail.com 
incluso trazabilidad. Nos veremos también enfrentados a la presión de la industria, encontrando nuevos nichos para sus productos, y ofreciéndolos como el estándar de cuidado[6]. Por último, la literatura científica también sufrirá presiones, relajando y acelerando los procesos editoriales con el objetivo de tener más artículos sobre el tema en boga. Debemos ser críticos e independientes al leer la información, contrastarla con la evidencia previa y el sentido común, y no perseguir todas las estrellas fugaces.

En lo técnico, centrar nuestro cuidado en los conocimientos de la fisiología, mejor evidencia científica y en la paciencia. No hay balas mágicas. En cuanto a la terapia ventilatoria, recordar los fundamentos de la ventilación protectora $(6-8 \mathrm{ml} / \mathrm{kg} \mathrm{IBW})$ [ARDSNET] [7], titular el PEEP según reclutabilidad, privilegiar una estrategia conservadora de fluidos (incluyendo no nutrir la primera semana)[8] y mínima sedación para mantener la sincronía paciente ventilador[7]-[10]. Si la $\mathrm{Pa} / \mathrm{FiO}_{2}$ baja de 150, aplicar precozmente prono[11], bloqueo neuromuscular en infusión [9] y esperar. Paciencia. No existe la no respuesta a PEEP o prono[11]. Existe pacientes poco reclutables (use PEEP 5-8) a muy reclutables (use PEEP 12 a 15), y en la mayoría basta con PEEP $10 \mathrm{cmH}_{2} \mathrm{O}[12]$,[13]. El beneficio del prono a veces se traduce en mejoría de la oxigenación, pero siempre protegerá al parénquima pulmonar del punto de vista mecánico. En cualquier caso, siempre debe mantener un volumen corriente cercano a 6 y no mayor a $8 \mathrm{ml} / \mathrm{kg}$ IBW[14]. Eso es protección pulmonar, y se puede aplicar desde Arica a Punta Arenas, incluso con ventiladores de anestesia.

Sin embargo, pese a todo este trabajo en equipo, y todo el armamentario farmacológico y terapéutico, habrá pacientes que fallecerán, siendo refractarios a nuestra terapia. En estos momentos difíciles en que nos enfrentamos a decisiones complejas entre la vida y la muerte, en la cual la medicina no siempre tiene respuestas claras, aparece nuestro rol del pastor y pasan a primar, así como hace 1.000 años, los principios éticos básicos que rigen nuestro actuar. Esto se resume en el principio de proporcionalidad terapéutica, que consiste en hacer lo humanamente posible, asegurándonos que el paciente y/o su familia entiendan la situación, conocer sus miedos y esperanzas, y decidir en conjunto el mejor camino a seguir.

En cada crisis también se abren oportunidades. Oportunidades para crear lazos, vínculos y redes de colaboración, tanto dentro del área sanitaria como con otros actores. Esta crisis es una oportunidad para reencantarnos con nuestra profesión, al poder enfocarnos de lleno en el cuidado de los pacientes e intentar marcar una diferencia. Por último, es una oportunidad para impulsar la innovación, la creación de soluciones, tanto tecnológicas, humanas y sociales.

Hacemos un llamado a anestesiólogos, intensivistas y a todo el equipo de salud a hacer bien lo que sabemos hacer bien. No olvidemos los fundamentos de los cuidados del paciente crítico, y "construyamos nuestra casa" sobre pilares sólidos. Hacemos un llamado a cuidar (física y emocionalmente) a nuestro equipo de trabajo. Hacemos un llamado a ser humildes, dar lo mejor de nosotros mismos y aprender de cada caso al que nos veamos enfrentados. Por último, hacemos un llamado a mantener en el centro de nuestro actuar a aquellos más vulnerables (los pacientes), y acompañarlos siempre, independiente del resultado de nuestras intervenciones.

\section{Referencias}

1. Ferguson ND, Cook DJ, Guyatt GH, Mehta S, Hand L, Austin P, et al.; OSCILLATE Trial Investigators; Canadian Critical Care Trials Group. High-frequency oscillation in early acute respiratory distress syndrome. N Engl J Med. 2013 Feb;368(9):795-805. https://doi. org/10.1056/NEJMoa1215554 PMID:23339639

2. Young D, Lamb SE, Shah S, MacKenzie I, Tunnicliffe W, Lall R, et al.; OSCAR Study Group.
High-frequency oscillation for acute respiratory distress syndrome. N Engl J Med. 2013 Feb;368(9):806-13. https://doi. org/10.1056/NEJMoa1215716 PMID:23339638

3. Zhu N, Zhang D, Wang W, Li $X$, Yang B, Song J, et al.; China Novel Coronavirus Investigating and Research Team. A Novel Coronavirus from Patients with Pneumonia in China, 2019. N Engl J Med. 2020 Feb;382(8):727-33. https://doi. org/10.1056/NEJMoa2001017 PMID:31978945
4. Wu C, Chen X, Cai Y, Xia J, Zhou X, Xu S, et al. Risk Factors Associated With Acute Respiratory Distress Syndrome and Death in Patients With Coronavirus Disease 2019 Pneumonia in Wuhan, China. JAMA Internal Medicine [Internet]. American Medical Association (AMA); 2020 Mar 13; https://doi.org/10.1001/ jamainternmed.2020.0994 PMID:32167524

5. Combes A, Hajage D, Capellier $G$, Demoule A, Lavoué $S$, Guervilly C, et al.; EOLIA Trial 
Group, REVA, and ECMONet.

Extracorporeal Membrane

Oxygenation for Severe

Acute Respiratory Distress

Syndrome. N Engl J Med.

2018 May;378(21):1965-75.

https://doi.org/10.1056/

NEJMoa1800385

PMID:29791822

6. Shehabi $Y$, Howe BD, Bellomo R, Arabi YM, Bailey M, Bass FE, et al.; ANZICS Clinical Trials Group and the SPICE III Investigators. Early Sedation with Dexmedetomidine in Critically III Patients. N Engl J Med. 2019 Jun;380(26):250617. https://doi.org/10.1056/ NEJMoa1904710 PMID:31112380

7. Brower RG, Matthay MA, Morris A, Schoenfeld D, Thompson BT, Wheeler A; Acute Respiratory Distress Syndrome Network. Ventilation with lower tidal volumes as compared with traditional tidal volumes for acute lung injury and the acute respiratory distress syndrome. N Engl J Med. 2000 May;342(18):1301-8. https://doi.org/10.1056/ NEJM200005043421801

\section{PMID:10793162}

8. Wiedemann HP, Wheeler AP, Bernard GR, Thompson BT, Hayden D, deBoisblanc B, et al.; National Heart, Lung, and Blood Institute Acute Respiratory Distress Syndrome (ARDS) Clinical Trials Network. Comparison of two fluidmanagement strategies in acute lung injury. N Engl J Med. 2006 Jun;354(24):2564-75. https:// doi.org/10.1056/NEJMoa062200 PMID:16714767

9. Papazian L, Forel JM, Gacouin A, Penot-Ragon C, Perrin G, Loundou A, et al.; ACURASYS Study Investigators. Neuromuscular blockers in early acute respiratory distress syndrome. N Engl J Med. 2010 Sep;363(12):1107-16. https://doi.org/10.1056/ NEJMoa1005372 PMID:20843245

10. Slutsky AS, Ranieri VM. Ventilator-induced lung injury. N Engl J Med. 2013 Nov;369(22):2126-36. https://doi.org/10.1056/ NEJMra1208707 PMID:24283226

11. Guérin C, Reignier J, Richard JC,
Beuret $P$, Gacouin A, Boulain

$T$, et al.; PROSEVA Study Group. Prone positioning in severe acute respiratory distress syndrome. N Engl J Med. 2013 Jun;368(23):2159-68. https://doi.org/10.1056/ NEJMoa1214103 PMID:23688302

12. Gattinoni $L$, Caironi $P, C$ Cressoni M, Chiumello D, Ranieri VM, Quintel $M$, et al. Lung recruitment in patients with the acute respiratory distress syndrome. N Engl J Med. 2006 Apr;354(17):1775-86. https:// doi.org/10.1056/NEJMoa052052 PMID:16641394

13. Bugedo G, Retamal J, Bruhn A. Does the use of high PEEP levels prevent ventilator-induced lung injury? Rev Bras Ter Intensiva. 2017 Apr-Jun;29(2):231-7. PMID:28977263

14. Bruhn A, Bugedo D, Riquelme F, Varas J, Retamal J, Besa $C$, et al. Tidal volume is a major determinant of cyclic recruitment-derecruitment in acute respiratory distress syndrome. Minerva Anestesiol. 2011 Apr;77(4):418-26. PMID:21483386 